\title{
Clasificación de los trastornos del sueño
}

\section{Classification of sleep disorders}

\author{
J. Gállego Pérez-Larraya, J.B. Toledo, E. Urrestarazu, J. Iriarte
}

\section{RESUMEN}

Los trastornos del sueño son una patología muy frecuente tanto aislada, propia como tal, o asociada a otros trastornos. Sin embargo, es una parte de la medicina relativamente nueva, dado que ha sido en los últimos 40 años cuando se ha trabajado realmente en ella, y se han producido los avances tanto diagnósticos como terapéuticos. Las clasificaciones de estas enfermedades han ido sufriendo cierta evolución, fijándose primero en los síntomas, y luego en las enfermedades. La nueva clasificación del 2005 vuelve a basarse en los síntomas. En ella se incluyen más de 90 enfermedades del sueño, y se intentan incluir tanto los síntomas, como las enfermedades propiamente del sueño y aquellas en las que los trastornos del sueño son fundamentales. Conocer y dominar esta completa clasificación es esencial para poder manejar adecuadamente estos pacientes. ción.

Palabras clave. Trastornos del sueño. Clasifica-

An. Sist. Sanit. Navar. 2007; 30 (Supl. 1): 19-36.

\begin{abstract}
Sleep disorders are frequent processes, both as a symptom associated with other diseases and as independent disorders. However, only in the last 4 decades has Sleep medicine gained its position among the medical specialties. In fact, it was only in these years that significant advances were obtained in the study of the etiology and treatment of these disorders. Similarly, the different classifications have been evolving over the years. First, they were based upon the clinical symptom; later on, more emphasis was given to the diseases. Finally, in 2005, the new classification was once again based on the symptoms. More than 90 disorders are listed in this latest classification, and an attempt is made to include the symptoms and the diseases of sleep, as well as those in which sleep disorders are fundamental. It is essential to have a clear idea of this complete classification of sleep disorders in order to deal with these patients appropriately.
\end{abstract}

Key words. Sleep disorders. Classification.
Unidad de Sueño. Clínica Universitaria, Universidad de Navarra. Pamplona

\author{
Correspondencia: \\ Jorge Iriarte \\ Unidad de Sueño \\ Clínica Universitaria \\ Universidad de Navarra \\ Avda. Pío XII, 36 \\ 31008 Pamplona \\ Fax. 948172294 \\ E-mail: jiriarte@unav.es
}




\section{INTRODUCCIÓN}

Los trastornos del sueño constituyen un grupo muy numeroso y heterogéneo de procesos. Hay muchísimas enfermedades que cursan con algún trastorno del sueño como uno más de sus síntomas. De hecho, es difícil encontrar alguna enfermedad que no altere en nada el sueño nocturno o la tendencia a dormir durante el día. Por ello, las clasificaciones han buscado clasificar los trastornos del sueño como enfermedades propias y no sólo como síntomas. Sin embargo, el modo de clasificar tales enfermedades se ha basado, en la mayoría de los casos, en el síntoma principal, y por ello se dividían en insomnios, hipersomnias, parasomnias, etc. Esto cambió en la primera Clasificación Internacional de Trastornos del Sueño ICSD-1 de 1990 y revisada en $1997^{1}$ (Tabla 1). Fue propuesta conjuntamente por la Asociación Americana (ASDA), Europea (ESRS), Japonesa (JSSR) y Latinoamericana (LASS). Quince años después de este intento la nueva clasificación ha vuelto al esquema tradicional, facilitando el identificar una enfermedad según su síntoma prin$\mathrm{cipal}^{2}$. Cada uno de los sistemas tiene ventajas e inconvenientes. En esta revisión presentamos la clasificación actual y las diferencias más importantes con respecto a la previa, incidiendo en las ventajas e inconvenientes, así como en las causas que han llevado a producir los cambios.

\section{CLASIFICACIONES DE LOS TRASTORNOS DEL SUEÑO RECIENTES}

\section{ICD-9 (Clasificación Internacional de Enfermedades, versión 9) ${ }^{3}$}

No incluye los trastornos del sueño como tales, recogiéndose las múltiples enfermedades del sueño en distintos apartados. De hecho sus códigos fueron los que se tomaron en la Clasificación Internacional de Trastornos del Sueño ICSD-1, propuesta en 1990.

\section{ICD-10 (Clasificación Internacional de Enfermedades, versión 10) ${ }^{4}$}

Dedica a los trastornos del sueño un capítulo propio. En el apartado G47 se incluyen los trastornos del sueño, distinguiendo entre insomnios, hipersomnias, alteración del ritmo sueño-vigilia, apnea

Tabla 1. Resumen de la ICSD-1.

\begin{tabular}{|c|c|c|c|}
\hline DISOMNIAS & PARASOMNIAS & $\begin{array}{l}\text { ALT ASOCIADAS A OTRAS } \\
\text { ENF }\end{array}$ & SD PROPUESTOS \\
\hline $\begin{array}{l}\text { 1-Intrínsecas } \\
\text {-Insomnio psicofisiológico } \\
\text { - Mala percepción del } \\
\text { sueño } \\
\text { - Insomnio idiopático } \\
\text { - Narcolepsia } \\
\text { - Hipersomnia idiopática } \\
\text {-SAOS } \\
\text {-MPP } \\
\text {-Sindrome de apneas cen- } \\
\text { trales } \\
\text { 2-Extrínsecas } \\
\text {-Secundario a: altitud, } \\
\text { alergia a alimentos, alco- } \\
\text { hol, fármacos, higiene } \\
\text { del sueño, ruido } \\
\text { 3-Alt. ritmo circadiano } \\
\text { - Jet-lag, ritmo irregular, } \\
\text { ciclo corto, ciclo largo, } \\
\text { trabajo en turnos }\end{array}$ & $\begin{array}{l}\text { 1-Alt. despertar } \\
\text {-Despertar confusional } \\
\text {-Sonambulismo } \\
\text { - Terrores nocturnos } \\
\text { 2-Alt. transición vigilia- } \\
\text { sueño } \\
\text { - Jactatio capitis } \\
\text {-Somniloquios } \\
\text { - Calambres nocturnos } \\
\text { 3-Parasomnias en REM } \\
\text {-Parálisis del sueño } \\
\text {-Pesadillas } \\
\text { - Alt de conducta en REM } \\
\text {-Parada sinusal en REM } \\
\text { 4-Otras parasomnias } \\
\text {-Bruxismo, Enuresis, Dis- } \\
\text { quinesia paroxística } \\
\text { nocturna, mioclonus, } \\
\text { ronquido primario, } \\
\text { hipoventilación congéni- } \\
\text { ta }\end{array}$ & $\begin{array}{l}\text { 1-Enf. Psiquiátricas } \\
\text {-Psicosis, alt pánico, alt } \\
\text { ansiedad, alt humor, } \\
\text { alcoholismo } \\
\text { 2-Enf. Neurológicas } \\
\text {-Insomnia fatal familiar } \\
\text {-Demencia } \\
\text {-Parkinsonismo } \\
\text {-Enf degenerativas } \\
\text {-Epilepsia, status del } \\
\text { sueño } \\
\text {-Cefalea relacionada al } \\
\text { sueño } \\
\text { 3-Enf. Médicas } \\
\text {-Isquemia cardiaca noc- } \\
\text { turna } \\
\text {-EPOC } \\
\text { - Asma relacionada al } \\
\text { sueño } \\
\text {-Reflujo gastroesofágico } \\
\text {-Ulcera gastroduodenal } \\
\text {-Fibromialgia } \\
\text {-Enf. del sueño }\end{array}$ & $\begin{array}{l}\text {-Hiperhidrosis del sueño } \\
\text {-Sd. de subvigilia } \\
\text {-Laringoespasmo } \\
\text {-Sueño largo } \\
\text { - Sueño corto } \\
\text { - Taquipnea } \\
\text { - Alucinaciones } \\
\text { - Atragantamiento } \\
\text { - Alt. en embarazo } \\
\text { - Alt. perimenstruales }\end{array}$ \\
\hline
\end{tabular}


del sueño, narcolepsia y cataplejia, "otros trastornos" del sueño, como el síndrome de Kleine-Levin, y trastornos del sueño noespecificados. Además en la sección F51 se introducen los trastornos del sueño noorgánicos, como el insomnio, hipersomnia, alteraciones del ritmo sueño-vigilia, sonambulismo, terrores nocturnos y pesadillas, además de un grupo de "otros" y otro grupo de "no-especificados" como, por ejemplo, las alteraciones del sueño emocionales.

\section{ICSD-1(Clasificación Internacional de Trastornos del Sueño) ${ }^{1}$}

Fue la propuesta conjunta de la Asociación Americana de Trastornos del Sueño (ASDA), Asociación Europea de Investigación en Sueño (ESRS), Sociedad Japonesa de Investigación en Sueño (JSSR) y la Sociedad Latinoamericana de Sueño (LASS). Esta clasificación comprendía tres ejes: el primero sobre el diagnóstico del proceso, el segundo sobre procedimientos diagnósticos en ese trastorno, y el tercero sobre las enfermedades médicas y psiquiátricas asociadas a ese trastorno. En cada eje se asignaba un código con letras y números intentando seguir en lo posible el ICD-9. Por ejemplo, se diría, que un paciente sufre un síndrome de apneas de sueño, moderado y crónico, siendo éste el diagnóstico final (eje A), confirmado por polisomnografía (eje B), y produciendo depresión reactiva e hipertensión arterial (eje C). En siglas sería 780.53 F23 (eje A), P2A (eje b), 300.4, 401.1 (eje C).

En el eje A se distinguían cuatro tipos de enfermedades del sueño: las disomnias, las parasomnias, las alteraciones asociadas a otras enfermedades, y una serie de síndromes propuestos como tales.

\section{CLASIFICACIÓN DE LOS TRASTORNOS DE SUEÑO ICSD-2}

La clasificación propuesta en 2005 vuelve al diagnóstico clínico tradicional, dando más peso al síntoma principal o a los tipos de trastornos que aparecen durante el sueño. Su objetivo es presentar una clasificación estructurada, racional y válida, que se base en la descripción clínica y científica y sea lo más compatible posible con las clasificaciones ICD-9 e ICD- $10^{2}$. Se centra en las enfermedades del sueño y no en los métodos diagnósticos. Sin embargo, sí sigue siendo también extensa en la descripción de cada uno de los trastornos del sueño, ya que incluye datos sobre cada proceso como son: otros nombres, características esenciales, características adicionales, demografía, patrón familiar, factores precipitantes y predisponentes, fisiopatología, hallazgos en la polisomnografía y otras pruebas, criterios diagnósticos, subtipos, datos no resueltos y direcciones futuras, diagnóstico diferencial y bibliografía. Por lo tanto, el libro de la clasificación más que una clasificación es un libro de referencia sobre los trastornos del sueño.

La principal diferencia con la ICSD-1 es el abandono de los 3 ejes de la clasificación previa siendo ahora de un solo eje. También elimina los números de los códigos de cada eje. Además desaconseja el uso de la palabra disomnia, por considerarla no adecuada para describir un síntoma que en realidad es una combinación de síntomas.

\section{INSOMNIO}

El insomnio es el más frecuente de todos los trastornos del sueño en la población general $^{5-7}$. Por insomnio se entiende la presencia de forma persistente de dificultad para la conciliación o el mantenimiento del sueño, despertar precoz (Fig. 1), o un sueño poco reparador, a pesar de disponer de condiciones adecuadas para el sueño; además, para el diagnóstico de insomnio, es necesario que tales dificultades produzcan en el paciente al menos una de las siguientes molestias diurnas: fatiga o sensación de malestar general, dificultad para la atención, concentración o memoria, cambios en el rendimiento socio-laboral (o escolar, en el caso de los niños), alteraciones del ánimo o del carácter, somnolencia, disminución de la energía, motivación o iniciativa, propensión a cometer errores en el trabajo o en la conducción de vehículos, síntomas somáticos como tensión muscular o cefalea, y preocupaciones, obsesiones o miedos en relación con el sueño ${ }^{811}$. 


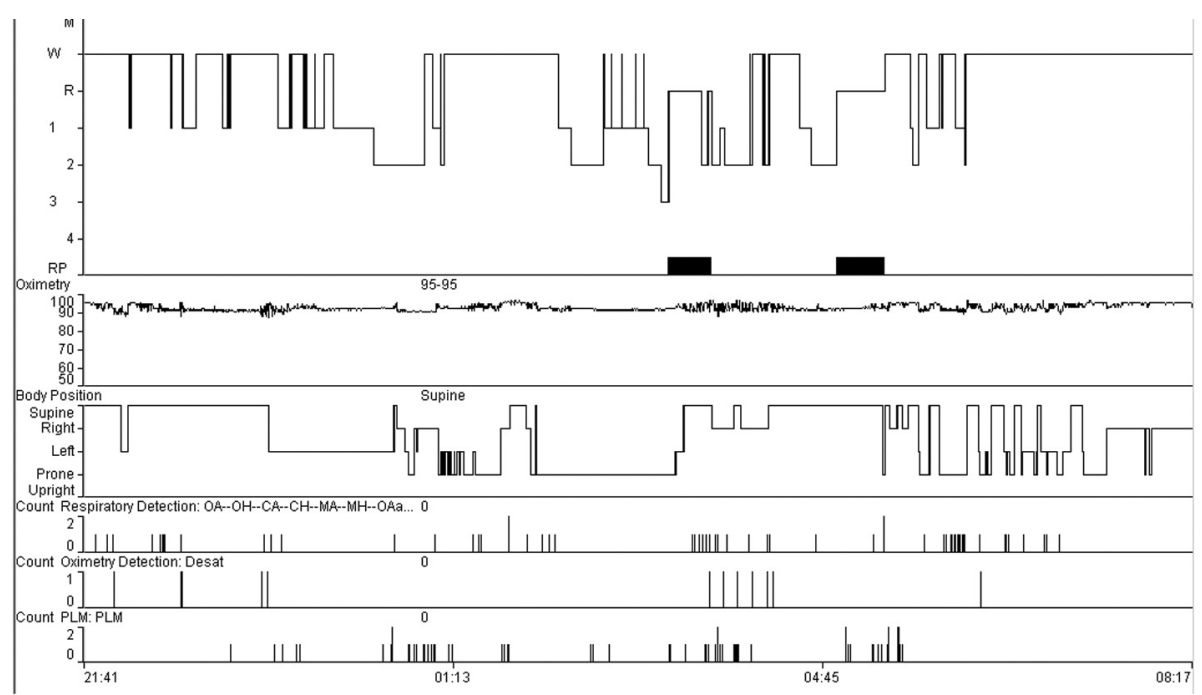

Figura 1. Hipnograma de un paciente con insomnio de conciliación, múltiples despertares nocturnos y baja eficiencia de sueño. $\mathrm{W}=$ vigilia; $\mathrm{R}=\mathrm{REM}$; $\mathrm{RP}=$ Periodo REM.

En los adultos, las quejas más habituales son la dificultad para la consolidación o el mantenimiento del sueño, y la existencia de largos periodos de vigilia durante la noche. Menos frecuentemente, las quejas de insomnio son referidas como sensación de sueño poco reparador o de baja calidad, a pesar de que el tiempo de sueño es percibido como normal o adecuado. En los niños es necesaria la descripción del sueño por un observador, y la dificultad para el sueño puede entenderse como una resistencia para irse a la cama o imposibilidad para dormir solo.

Una primera distinción diferencia entre insomnio primario (aislado, problema propiamente) y secundario (manifestación secundaria a una enfermedad orgánica, mental, otro trastorno primario del sueño, o al consumo o exposición a determinadas sustancias, drogas o fármacos $)^{12}$. Como el insomnio secundario es parte de otros procesos, la clasificación internacional hace hincapié en los trastornos propiamente del sueño. Entre ellos distingue varios tipos.

Insomnio agudo. La aparición de los síntomas definitorios de insomnio guarda una clara relación con un acontecimiento estresante. Éste puede ser de carácter psicológico, psicosocial, interpersonal o del entorno, siendo algunos ejemplos los cambios o conflictos en las relaciones interpersonales, el duelo, el diagnóstico de una enfermedad o el traslado a otra ciudad. Existen también factores estresantes con un sentido emocional positivo. La duración de este tipo de insomnio suele ser inferior a los 3 meses, resolviéndose cuando desaparece el acontecimiento estresante o cuando el sujeto logra adaptarse a él.

Su prevalencia exacta es desconocida, aunque se estima en torno al $15-20 \%$ en la población adulta general. Es más frecuente en mujeres que en varones, y aunque puede presentarse a cualquier edad, aparece con más frecuencia en adultos mayores que en adultos jóvenes o niños.

Insomnio psicofisiológico. Se define así a la dificultad condicionada para dormirse y/o extrema facilidad para despertarse del sueño, durante un periodo superior al mes, expresada por al menos una de las siguientes condiciones: ansiedad o excesiva preocupación por el sueño; dificultad para quedarse dormido al acostarse o en siestas planificadas, pero no en otros 
momentos del día en los que se llevan a cabo tareas monótonas y en los que no hay intención de dormirse; hiperactividad mental, pensamientos intrusivos o incapacidad para cesar la actividad mental, que mantienen al sujeto despierto; mejoría del sueño fuera del entorno y dormitorio habituales; excesiva tensión somática en la cama, que incapacita la relajación e impide conciliar el sueño ${ }^{13,14}$. Afecta aproximadamente al $1-2 \%$ de la población general, y predomina en el sexo femenino, y en grupos de edad de adolescentes y adultos jóvenes.

Insomnio paradójico. Se denomina también pseudoinsomnio o mala percepción del sueño. La principal característica es una queja de insomnio grave, sin que pueda objetivarse un trastorno de tal magnitud mediante la realización de pruebas diagnósticas como la polisomnografía. Existe por tanto una discordancia entre los resultados de la polisomnografía y las quejas subjetivas de los pacientes, quienes refieren dormir nada o muy poco todas las noches y de forma crónica. Constituye el $5 \%$ de todos los casos de insomnio, y es más frecuente en mujeres.

Insomnio idiopático. Se trata de un insomnio que aparece durante la infancia o durante los primeros años de la juventud, sin que se pueda reconocer un factor desencadenante o una causa que lo justifique. Es un insomnio crónico, que cursa de forma persistente, sin periodo alguno de remisión. Aunque su inicio se establezca en la infancia o juventud, no suele ser hasta muchos años después cuando se reconoce y se reclama la ayuda de un especialista.

Su prevalencia es, aproximadamente, del $0,7 \%$ en adolescentes y del $1 \%$ en adultos jóvenes, desconociéndose en grupos de mayor edad. También es posible el cuadro opuesto, es decir, que en vez de sentir insomnio sientan dormir bien cuando en realidad hay insomnio ${ }^{15}$.

Insomnio debido a trastornos mentales. Se define así al insomnio que dura al menos un mes y que está causado por una enfermedad mental subyacente (guarda una relación temporal, en la mayoría de los casos, con un trastorno mental diagnosti- cado según los criterios de la DSSMD). El insomnio no es sino un síntoma más de la enfermedad mental, aunque en muchas ocasiones puede ser más intenso que el habitualmente asociado al trastorno mental subyacente, requiriendo entonces un tratamiento independiente del proceso de base.

Es el insomnio más frecuentemente diagnosticado entre los pacientes que acuden a consultas especializadas en trastornos del sueño. Aproximadamente un 3\% de la población general tiene síntomas compatibles con este diagnóstico.

Insomnio debido a una inadecuada higiene del sueño. Es el insomnio asociado a actividades diarias que necesariamente impiden una adecuada calidad del sueño y mantenerse despierto y alerta durante el día. Un elemento común a esas actividades es que son prácticas que están bajo el control de la voluntad del sujeto. Pueden ser prácticas que conllevan un estado de hiperalerta o hipervigilia (el consumo rutinario y antes de acostarse de alcohol o cafeína; el desarrollo, también antes de acostarse, de intensas actividades mentales, físicas o emocionales; o condiciones inapropiadas de luz, ruido, etc., o prácticas que impiden una correcta estructuración el sueño -siestas frecuentes durante el día, grandes variaciones en las horas de acostarse o levantarse, pasar mucho tiempo en la cama-, etc. Su prevalencia se estima en torno al 1-2\% en los adolescentes y adultos, y constituye alrededor de un 5-10\% de los insomnios.

Insomnio debido a fármacos o tóxicos. Es la interrupción o supresión del sueño relacionada con el consumo de fármacos, drogas, cafeína, alcohol, comida, o con la exposición a un tóxico ambiental. El trastorno del sueño puede aparecer durante periodos de consumo o exposición, o también durante periodos de retirada o abstinencia. Afecta aproximadamente al $0,2 \%$ de la población general, y al 3,5\% de los sujetos que consultan por problemas de sueño.

Insomnio debido a problemas médicos. Es el causado por una enfermedad orgánica coexistente o por otra alteración fisiológica, de modo que se inicia con 
dicho problema médico y varía según las fluctuaciones o cambios en su curso. Afecta al $0,5 \%$ de la población general, siendo más frecuente en personas de edad avanzada. Alrededor de un $4 \%$ de los pacientes que son valorados en Unidad de Sueño presentan este tipo de insomnio.

\section{TRASTORNOS RESPIRATORIOS}

En este grupo se incluyen aquellos trastornos del sueño que se caracterizan por una alteración de la respiración durante el sueño. Los síndromes de apnea central del sueño incluyen aquellos trastornos en los que el movimiento respiratorio está disminuido o ausente de una manera intermitente o cíclica, debido a una disfunción cardíaca o del sistema nervioso central. En el síndrome de apneas obstructivas del sueño se produce una obstrucción al flujo del aire en la vía aérea, de manera que en presencia de movimientos respiratorios, a menudo enérgicos para intentar superar la obstrucción, hay una inadecuada ventilación (Fig. 2). Además, la segunda Clasificación Internacional de los Trastornos del Sueño distingue algunos otros tipos, más infrecuentes, pero importantes en el diagnóstico diferencial y también por requerir enfoques terapéuticos diversos.

Apnea central primaria. Es un trastorno de etiología desconocida, que se carac- teriza por episodios recurrentes de cese de la respiración durante el sueño, sin que se asocie a la apnea ningún movimiento ni esfuerzo respiratorio ${ }^{16}$. En la forma congénita es conocida como la maldición de Ondina $^{17}$. Los pacientes que tienen apnea central primaria suelen tener una presión arterial de $\mathrm{CO}_{2}$ por debajo de $40 \mathrm{mmHg}$ en vigilia. Se sospecha que el principal mecanismo fisiopatológico de este trastorno es una elevada respuesta ventilatoria a los niveles de $\mathrm{CO}_{2}$. La repetición de estos episodios de apnea central a lo largo de la noche produce una fragmentación del sueño, despertares frecuentes y rápidos tras las pausas de respiración, y excesiva somnolencia diurna. Para su diagnóstico es necesario además el registro mediante polisomnografía de 5 ó más apneas centrales por hora de sueño.

Patrón respiratorio de Cheyne Stokes. Este patrón respiratorio se caracteriza por la aparición de al menos 10 apneas e hipopneas de origen central, por hora de sueño, produciéndose en estas últimas una fluctuación progresiva del volumen tidal, siguiendo un patrón creciente-decreciente. Como en otras formas de apnea central, durante las pausas de respiración no se produce ningún esfuerzo ventilatorio. Habitualmente estos episodios se acompañan de despertares frecuentes, con la consiguiente desestructuración del

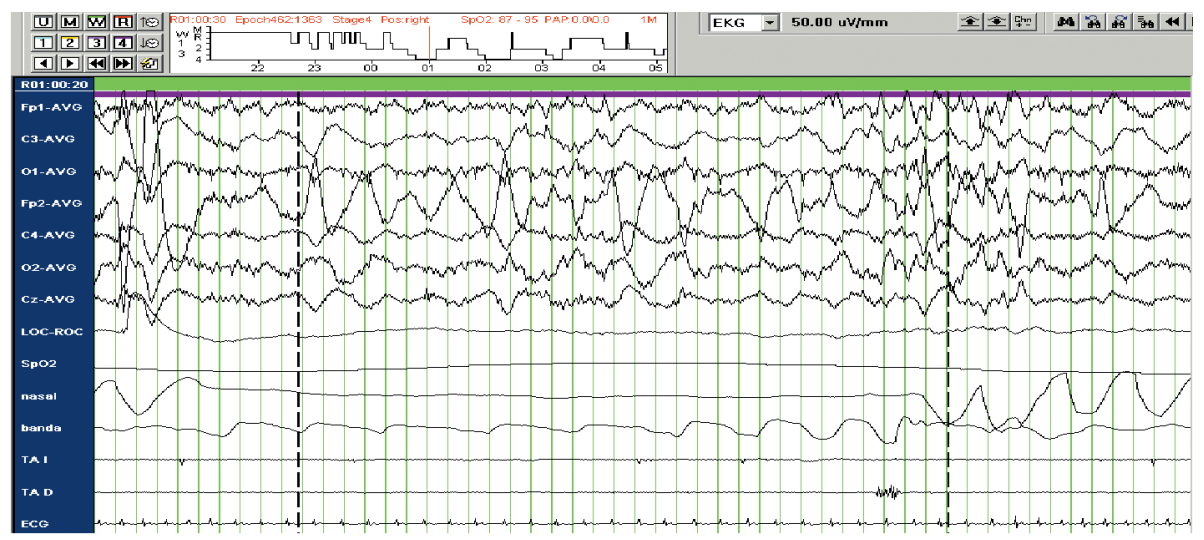

Figura 2. Registro polisomnográfico de una apnea obstructiva. El flujo se hace plano (nasal) mientras la banda respiratoria sigue moviéndose (banda). El paciente está en fase 4 de sueño. 
sueño. Este patrón de sueño se observa fundamentalmente durante las fases de sueño no-REM, y se resuelve parcial o completamente durante la fase REM. Los factores predisponentes más importantes son la insuficiencia cardiaca congestiva, el infarto cerebral y probablemente también la insuficiencia renal. El tratamiento es por tanto el de estas causas, pudiendo emplearse también el oxígeno a bajo flujo como tratamiento sintomático.

Otro tipo de apnea central es la que aparece en el periodo normal de adaptación a grandes altitudes, especialmente las superiores a 4.000 metros, y la apnea central del sueño secundaria a fármacos o sustancias, que se observa fundamentalmente en pacientes que siguen un tratamiento prolongado con opioides, como metadona, morfina y codeína.

Síndrome de apnea obstructiva del sueño (SAOS). Consiste en la presencia, durante el sueño, de episodios repetidos de obstrucción, completa en el caso de las apneas y parcial en el caso de las hipopneas, al flujo aéreo en la vía aérea alta ${ }^{18}$. Estos episodios provocan con frecuencia una reducción de la saturación de oxígeno. Por definición, las apneas e hipopneas deben durar un mínimo de 10 segundos, y no es infrecuente que se prolonguen incluso durante un minuto o más. Pueden ocurrir en cualquier fase del sueño, aunque lo más frecuente es que lo hagan en las fases 1 y 2 , y en la fase REM, produciéndose típicamente las mayores desaturaciones en esta última fase $\mathrm{e}^{19,20}$. Un signo frecuentemente asociado son los ronquidos muy intensos, que suelen producirse entre las apneas. Como consecuencia de las apneas e hipopneas se producen microdespertares, que desestructuran el sueño y hacen que los pacientes se levanten por la mañana cansados, sin sensación de sueño reparador, y tiendan a quedarse dormidos con facilidad durante el día. La actividad de los músculos dilatadores de la faringe, encargados de abrir la vía aérea superior se reduce durante el sueño. Una disminución sobreañadida del área de la vía aérea superior, debida a un excesivo volumen de tejidos blandos circundantes, a peculiaridades de la anatomía craneofacial, a la postura en decúbito supino, al consumo de alcohol o al empleo de fármacos miorrelajantes, entre otros, facilita la obstrucción al paso del aire y el desarrollo del síndrome de apnea e hipopnea obstructiva del sueño ${ }^{21}$. El tratamiento de este síndrome debe ser etiológico, tratando de corregir aquellos factores que provocan el cierre de la vía aérea. Como tratamiento sintomático se puede emplear una mascarilla de presión positiva continua (CPAP) durante el sueño ${ }^{22}$.

Hipoventilación alveolar central. También es conocida como hipoventilación del obeso. Se caracteriza por la desaturación arterial de oxígeno secundaria a un descenso en la ventilación alveolar, que tiene lugar en pacientes sin enfermedades pulmonares primarias, malformaciones esqueléticas o trastornos neuromusculares que afecten a la capacidad de ventilación. Aparecen desaturaciones de oxígeno en REM, sin apneas concluyentes. Además de la forma idiopática también puede ser secundaria a las patologías citadas.

Hipoventilación alveolar central congénita. Se produce por un fallo en el control central de la respiración. La hipoventilación se inicia en la infancia, empeora durante el sueño, y no puede explicarse por ninguna enfermedad pulmonar primaria, neurológica ni metabólica.

\section{HIPERSOMNIAS}

Esta sección incluye un grupo de enfermedades caracterizadas fundamentalmente por somnolencia diurna, que no es atribuible a ninguna dificultad para el sueño nocturno ni a cambios en el ritmo circadiano. Se entiende por somnolencia diurna la incapacidad para mantenerse despierto y alerta durante la mayoría de las situaciones del día. Ésta ocurre con mayor probabilidad en situaciones monótonas que no requieren una participación activa. Muchas veces conllevan un aumento en el tiempo total de sueño diario, sin que ello suponga una sensación de sueño reparador. En otros casos conlleva un comportamiento automático que no impide realizar actividades durante el estado de somnolencia.

La severidad de la somnolencia diurna puede cuantificarse subjetivamente median- 
te la escala Epworth Sleepines Scale, y objetivamente mediante los datos del Test de Latencias Múltiples (latencia de entrada en sueño y número de entradas en REM en cinco siestas consecutivas, separadas 2 horas, durante un día) y el Test de Mantenimiento de la Vigilia (latencia de sueño y entrada en REM o no en cinco intentos consecutivos, separados 2 horas, a lo largo de un día, de mantener la vigilia en condiciones favorecedoras del sueño (cama, silencio, obscuridad)).

En la segunda Clasificación Internacional de los Trastornos del Sueño se incluyen en este grupo las siguientes entidades:

Narcolepsia con cataplejia o síndrome de Gelineau. Se caracteriza por una excesiva somnolencia diurna y cataplejia. Muchos de sus síntomas se deben a una inusual propensión para pasar, y de forma muy rápida, del estado de alerta a la fase de sueño REM, y para experimentar eventos del sueño REM disociados. El primer síntoma en aparecer, y el más incapacitante, es la somnolencia diurna; se caracteriza por siestas repetidas o entradas repentinas en sueño durante el día. Típicamente, los pacientes duermen durante un periodo corto de tiempo y se despiertan descansados, pero a las 2 ó 3 horas vuelven a presentar somnolencia, repitiéndose este patrón numerosas veces a lo largo del día. La somnolencia aparece más fácilmente en situaciones monótonas que no requieren una participación activa, aunque pueden aparecer ataques repentinos e incontrolables de sueño en circunstancias inusuales como caminando, conduciendo, comiendo, etc. El otro síntoma característico, la cataplejia, se caracteriza por una pérdida brusca del tono muscular, provocada por emociones fuertes y habitualmente positivas. Puede ser localizada o afectar a todos los grupos musculares del esqueleto, salvo los músculos respiratorios, que no se afectan nunca. Su duración suele ser de segundos o pocos minutos, y la recuperación es inmediata y completa. Estímulos repetidos pueden provocar un estatus, pudiendo prolongarse la crisis incluso durante una hora. Otros síntomas que pueden aparecer son la parálisis del sueño, alucinaciones hipnagógicas, sueño desorganizado o conductas automáticas. La parálisis del sueño se caracteriza por una incapacidad generalizada y transitoria para moverse o hablar; ocurre fundamentalmente durante la transición sueño-vigilia; y el paciente recupera el control de su musculatura en unos pocos minutos. Las alucinaciones hipnagógicas consisten en sueños muy vivenciados que ocurren especialmente al inicio del sueño, y que cursan con fenómenos visuales, táctiles y auditivos.

Existe una fuerte asociación de este trastorno con el HLA (DQB1 *0602 ó DR2) y al déficit de hipocretinas del hipotálamo posterior $^{23,24}$. El diagnóstico de sospecha es clínico, y debe confirmarse mediante el Test de Latencia Múltiple.

Existen la narcolepsia sin cataplejia, caracterizada por siestas repetidas y reparadoras durante el día con un sueño nocturno conservado, y la narcolepsia debida a alteraciones médicas o neurológicas, como en casos de tumores o placas desmielinizantes que afectan al hipotálamo, síndromes paraneoplásicos con anticuerpos anti-Ma2 ${ }^{25}$.

Hipersomnia recurrente. Se trata de una entidad poco frecuente, habiéndose descrito en la literatura médica sólo unos 200 casos, con ligero predominio en el sexo masculino. Su principal paradigma es el síndrome de Kleine-Levin ${ }^{26}$. Se caracteriza por la aparición de episodios de hipersomnia con una frecuencia que oscila entre 1 y 10 veces por año. Los episodios pueden durar desde pocos días hasta varias semanas. Frecuentemente vienen precedidos por pródromos consistentes en fatiga o cefalea, de unas pocas horas de duración. Durante los episodios de hipersomnia, los pacientes pueden dormir de 16 a 18 horas al día, despertándose o levantándose únicamente para comer. No es infrecuente que los pacientes vean incrementado su peso durante los episodios. Otras alteraciones del comportamiento, como hiperfagia, hipersexualidad, irritabilidad o agresividad, y también cognitivas o mentales, como confusión, desrealización, alucinaciones, etc., pueden manifestarse durante los periodos de hipersomnia. No obstante, la confluencia de todos estos síntomas es una excepción, y lo más habitual es que la hipersomnia se presente de forma aislada. El sueño y el 
comportamiento interepisódico son absolutamente normales.

Hipersomnia idiopática con sueño prolongado. Se caracteriza por una somnolencia excesiva, constante y diaria durante al menos tres meses. El sueño nocturno se prolonga durante unas 12-14 horas, con ningún o muy pocos despertares. Durante el día el paciente puede realizar siestas de 3 ó 4 horas de duración, sin que resulten reparadoras. Los pacientes aquejados de esta enfermedad tienen además una gran dificultad para despertarse tanto del sueño nocturno como de las siestas.

Hipersomnia idiopática sin sueño prolongado. Se distingue de la anterior en que en este caso el sueño nocturno suele tener una duración entre 6 y 10 horas, no excediendo este límite. No se acompaña de cataplejia, aunque los pacientes pueden tener a veces dificultad para despertarse del sueño nocturno y también de las siestas.

Sueño insuficiente inducido por el comportamiento. Consiste en la somnolencia secundaria a una deprivación crónica del sueño, voluntaria, pero no buscada directamente, derivada de comportamientos que impiden alcanzar la cantidad de sueño necesario para mantener un adecuado nivel de vigilia y alerta (obligada por circunstancias como el trabajo, niños pequeños en casa).

Otros tipos de hipersomnia. Otros tipos de hipersomnia son la hipersomnia debida a una enfermedad médica (enfermedades neurológicas como la enfermedad de Parkinson y lesiones del hipotálamo, enfermedades endocrinológicas como el hipotiroidismo, trastornos metabólicos como la encefalopatía hepática o la insuficiencia renal, etc.), la hipersomnia secundaria al consumo de fármacos o drogas, y la hipersomnia no debida a ninguna condición fisiológica ni al consumo de sustancias sino a un trastorno mental, y la mala percepción de hipersomnia (también conocida como pseudohipersomnia) ${ }^{15}$.

\section{ALTERACIONES DEL RITMO CIRCADIANO}

Para obtener un sueño óptimo, reparador o de buena calidad, el tiempo dedica- do al mismo debería coincidir con el ritmo circadiano biológico del sueño de cada individuo. En la segunda Clasificación Internacional de los Trastornos del Sueño se describen las siguientes alteraciones del ritmo circadiano:

Síndrome de la fase del sueño retrasada. Se caracteriza, como su propio nombre indica, por un retraso -habitualmente mayor de dos horas- en los tiempos de conciliación del sueño y despertar, en relación con los horarios convencionales o socialmente aceptados. Los individuos afectados por esta entidad tienen una práctica imposibilidad para dormirse y despertarse a una hora razonable, haciéndolo más tarde de lo habitual. La estructura del sueño es normal, destacando únicamente en los estudios polisomnográficos un importante alargamiento de la latencia del sueño o el tiempo que tardan en dormirse los pacientes. Estos tienen con frecuencia problemas socio-laborales, ya que sus horas de mayor actividad suelen ser las de la noche. En estos individuos están también retrasados otros ciclos biológicos circadianos, como son el de la temperatura y el de la secreción de melatonina.

Parece existir un componente genético, habiéndose demostrado asociaciones entre polimorfismos en algunos genes (hPer3, HLA, Clock,...) y el retraso en la fase del sueño. Es más frecuente entre adolescentes y adultos jóvenes, siendo su prevalencia del $7-16 \%$. Aunque se han descrito casos de inicio en la infancia, la edad media de inicio de los síntomas son los 20 años.

Síndrome de la fase del sueño adelantada. Es menos frecuente que el síndrome de la fase retrasada. Los periodos de conciliación del sueño y de despertar son muy tempranos o precoces con respecto a los horarios normales o deseados. Los sujetos que padecen este síndrome suelen quejarse de somnolencia durante la tarde y tienen tendencia a acostarse muy pronto, y se despiertan espontáneamente también muy pronto por la mañana. Cuando se acuestan muy tarde, por factores exógenos, sufren un déficit de sueño, ya que su ritmo circadiano les despierta igualmente pronto. No se conoce su prevalencia, pero 
se estima en torno al $1 \%$ en los adultos y ancianos, y aumenta con la edad (probablemente porque con la edad se acorta el ritmo circadiano). Afecta a ambos sexos por igual.

Ritmo sueño-vigilia irregular. Se caracteriza por la ausencia de un ritmo circadiano sueño-vigilia claramente definido. Tal ritmo está tan desestructurado, o es tan caótico, que los periodos de sueño y vigilia son absolutamente variables a lo largo de las 24 horas del día. Aunque el tiempo de sueño total es normal, los pacientes muestran síntomas y signos de insomnio o somnolencia dependiendo de la hora del día.

Ritmo sueño-vigilia libre. El ritmo sueño-vigilia no coincide con el patrón típico de 24 horas. Lo más frecuente es que el ritmo sea más largo que el típico de 24 horas, pero también puede ser más corto o variable. Esta alteración es muy frecuente en sujetos completamente ciegos, debido a la pérdida del estímulo luminoso como marcapasos más importante para un correcto ritmo circadiano. Se presenta también en sujetos con retraso mental, demencia y trastornos psiquiátricos.

Jet Lag o alteración del sueño por viajes con cambio de huso horario. Se caracteriza por un desajuste entre el ritmo circadiano endógeno de sueño-vigilia y el patrón exógeno de sueño-vigilia de una zona geográfica determinada, derivado de un desplazamiento de gran distancia -que conlleva el cruce de varios meridianos terrestres- en un corto periodo de tiempo. En estos desplazamientos, el ritmo circadiano permanece anclado en el horario del punto de partida, y se ajusta lentamente al horario del punto de llegada. Se trata de un proceso autolimitado, que puede durar hasta 6 días. La gravedad de sus síntomas (insomnio, somnolencia, astenia, bajo rendimiento intelectual, etc.) depende del número de meridianos terrestres que se cruzan, y parece ser mayor en sujetos de edad más avanzada. En los desplazamientos hacia el Este (la alteración es similar a un retraso del sueño) el desajuste suele ser mayor que en los desplazamientos inversos, en los que predomina una altera- ción similar al síndrome de adelanto de la fase de sueño.

Alteración del trabajador nocturno. En esta alteración del ritmo circadiano, los síntomas de insomnio o hipersomnia son secundarios a jornadas o turnos laborales que se solapan con el periodo normal del sueño, permaneciendo el trabajador alerta en un momento inadecuado de su ciclo sueño-vigilia. No sólo se presenta en trabajadores que tienen turnos nocturnos o de madrugada, sino también en aquellos con rotación de los turnos; estos últimos necesitan un tiempo determinado para adaptar su ciclo sueño-vigilia a la nueva situación. Habitualmente, todas estas condiciones de trabajo conllevan una reducción de las horas de sueño con un desajuste del ritmo circadiano de sueño-vigilia.

Alteración del ritmo circadiano debida a un proceso médico. Es el trastorno del ritmo circadiano en relación con una enfermedad. En general, el ingreso en un hospital, especialmente en sujetos de edad avanzada, es una condición suficiente para producir un cambio del ritmo sueño-vigilia. Los ingresos prolongados, las intervenciones quirúrgicas, el empleo de fármacos sedantes o hipnóticos, las estancias en unidades de cuidados intensivos (en las que hay una actividad continuada las 24 horas del día, sin apenas interrupción de estímulos sonoros y luminosos), y los procesos metabólicos e infecciosos, son factores que facilitan esta alteración.

\section{PARASOMNIAS}

Las parasomnias son trastornos de la conducta o comportamientos anormales que tienen lugar durante el sueño ${ }^{27}$. En la segunda edición de la Clasificación Internacional de los Trastornos del Sueño se dividen en tres grupos: parasomnias del despertar, parasomnias asociadas al sueño REM, y otras parasomnias. En muchos casos hay datos sobre la influencia genética ${ }^{28}$.

\section{Parasomnias del despertar ${ }^{29}$}

Despertar confusional. Se conoce también como "borrachera del despertar". Se caracteriza por la aparición de un cuadro confusional al despertarse del sueño. 
Los individuos que sufren este trastorno se despiertan desorientados en tiempo y en espacio, con bradipsiquia y con una marcada disminución de la atención y de la respuesta a los estímulos; habitualmente suelen presentar también cierto grado de amnesia anterógrada o retrógrada. El comportamiento durante el despertar confusional puede ser apropiado o, por el contrario, el sujeto puede mostrarse agresivo y violento, consigo mismo o con el acompañante. Los episodios confusionales pueden durar desde algunos minutos, lo más frecuente, hasta varias horas.

Su prevalencia es mayor en los niños y en los adultos jóvenes, y habitualmente desaparecen con la edad.

Sonambulismo. Este trastorno consiste en el desarrollo, durante las fases de sueño profundo o delta (generalmente en la primera mitad del tiempo de sueño), de una secuencia de comportamientos complejos que habitualmente incluyen el caminar. Antes de comenzar a caminar, los individuos pueden sentarse en la cama y mirar alrededor de una forma confusa. Además, pueden llevar a cabo otras conductas aprendidas durante la vigilia, e incluso adoptar comportamientos agresivos. Los ojos suelen permanecer abiertos y con sensación de asombro. Durante los episodios, los sujetos se encuentran profundamente dormidos, siendo difícil despertarles del sueño; cuando esto se consigue, suelen encontrarse confusos, y con amnesia de lo ocurrido. Es más frecuente en la infancia, con una prevalencia aproximada de un $17 \%$, y tiende a desaparecer con la edad, siendo la prevalencia en adultos del $4 \%$. No existe un tratamiento etiológico, siendo necesario el adoptar medidas que eviten accidentes fortuitos.

Terrores nocturnos. Se caracterizan por la aparición súbita y durante las fases de sueño profundo, en la primera mitad de la noche, de episodios de llanto o grito inesperados, con una expresión facial de miedo o terror intensos, y que se acompañan de una importante descarga autonómica, con taquicardia, taquipnea, diaforesis, etc. Habitualmente el sujeto permanece sentado en la cama durante los episodios, profundamente dormido y sin responder a los estímulos externos; si se le despierta suele encontrarse confuso y desorientado, sin recordar lo que estaba sucediendo. Este trastorno es más propio de la infancia, donde la prevalencia es del 1-6,5\%, aunque también puede aparecer en edades más avanzadas.

\section{Parasomnias asociadas al sueño REM $^{30,31}$}

Trastorno de conducta del sueño $\boldsymbol{R E M}$. Este trastorno se caracteriza por la aparición de conductas anómalas durante el sueño REM. En lugar de presentar una pérdida completa del tono muscular propia de la fase REM del sueño, los sujetos con este trastorno suelen realizar movimientos, habitualmente violentos como puñetazos o patadas, que se corresponden con la actividad motora propia de la ensoñación en curso $^{32-34}$ (Fig. 3). Como consecuencia de tales movimientos, los sujetos pueden autolesionarse o lesionar al compañero de cama de forma fortuita. Otros tipos de conducta menos agresivos son accesos de risa, gritos, movimientos de gesticulación o de rascado, etc. Durante los episodios los sujetos suelen permanecer con los ojos cerrados. Típicamente, al final de cada episodio, el sujeto se despierta bruscamente, refiriendo una ensoñación muy vivenciada y coherente con los movimientos que ha desarrollado durante la fase de sueño REM. El trastorno de conducta asociado al sueño REM afecta predominantemente a varones por encima de los 50 años, y se ha asociado a sinucleinopatías $^{35,36}$.

Para su diagnóstico no basta la fenomenología clínica, sino que es preciso el registro polisomnográfico con electromiografía, para objetivar la ausencia de atonía permanente en la fase REM y el aumento de las fases 3 y 4 del sueño No-REM, y para descartar la existencia de actividad epileptiforme. El tratamiento farmacológico de elección es clonazepam, siendo además aconsejable retirar del entorno todos aquellos objetos con los que se pueda lesionar el sujeto.

Parálisis del sueño aislada. Consisten en una incapacidad para hablar y realizar cualquier movimiento voluntario con 


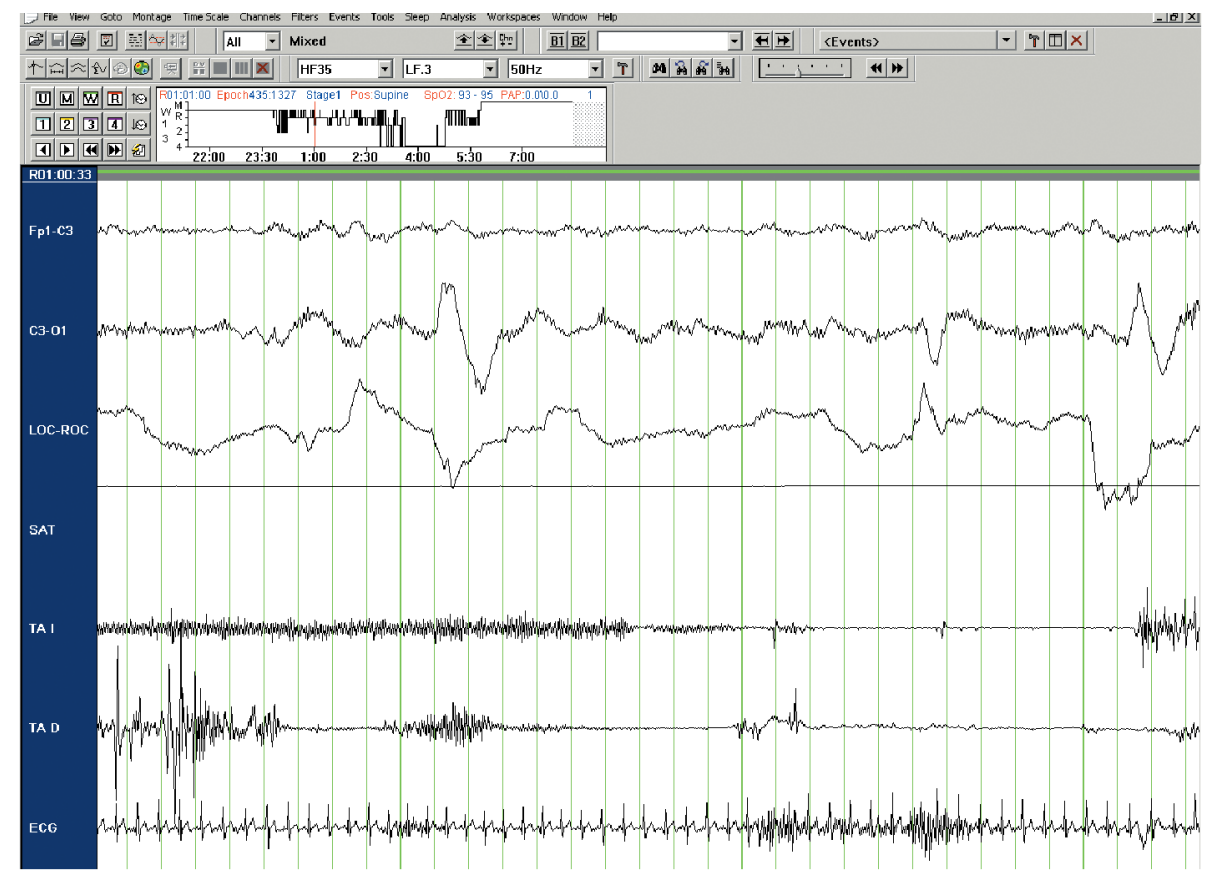

Figura 3. Registro polisomnográfico en trastorno de conducta de sueño REM. Es REM (movimientos oculares en el electrooculograma LOC-ROC) a la vez que hay aumento de tono muscular en los tibiales (TAD, TAI).

la cabeza, el tronco o las extremidades, debido a una pérdida completa del tono muscular. Los episodios suelen suceder al inicio de las fases de sueño REM o en la transición sueño-vigilia. Pueden durar unos segundos o incluso minutos. Durante los mismos, especialmente si son los primeros episodios, el sujeto puede experimentar intensa sensación de ansiedad, y puede también tener alucinaciones.

Pesadillas. Son ensoñaciones muy vivenciadas, de contenido desagradable, y que producen una importante sensación de miedo en el sujeto, llegando a despertarle en muchas ocasiones. Ocurren durante la fase REM del sueño, y a diferencia de los terrores nocturnos, predominan en la segunda mitad de la noche y el sujeto suele recordar y es consciente de lo sucedido cuando se despierta. Aunque es muy frecuente en la infancia, los adultos pueden tener también pesadillas de forma ocasional.

Otras parasomnias. Se incluyen los trastornos disociativos del sueño, la enuresis, la catatrenia o quejido nocturno, el síndrome de explosión cefálica, las alucinaciones del sueño y el síndrome de comida nocturna ${ }^{37}$.

\section{MOVIMIENTOS ANORMALES RELACIONADOS CON EL SUEÑO}

La alteración del sueño nocturno o las quejas de fatiga y somnolencia diarias son requisitos fundamentales para incluir en esta sección entidades que cursan con movimientos anormales ${ }^{38,39}$. La clasificación de movimientos anormales incluye los relacionados con el sueño ${ }^{40}$. Otro 
aspecto diferente, aunque relacionado, son los trastornos del sueño asociados a enfermedades del control motor ${ }^{41}$. Los principales trastornos del sueño que cursan con movimientos anormales, que se describen en la segunda Clasificación Internacional de los Trastornos del Sueño son los siguientes:

Síndrome de piernas inquietas. Es un trastorno del sueño caracterizado por una necesidad imperiosa e irresistible de mover las piernas ${ }^{42-44}$ (Fig. 4). Con mucha frecuencia, aunque no siempre, se acompaña de incómodas e incluso dolorosas parestesias en el interior de las piernas. La necesidad de mover las piernas y las posibles parestesias acompañantes empeoran con el reposo (en decúbito o en sedestación), y suelen mejorar, de forma prácticamente inmediata, caminando o moviendo las piernas. Suele ser peor por las tardes y las noches, mientras que su intensidad dis- minuye considerablemente por las mañanas. Aparece en la transición vigilia-sueño, cuando el paciente está todavía despierto, dificultando la conciliación del sueño al principio de la noche o tras los despertares nocturnos. Aunque, como su propio nombre indica, afecta predominantemente a las extremidades inferiores, los síntomas pueden presentarse también en los miembros superiores.

Su prevalencia es de aproximadamente el 5-10\%, siendo ligeramente más frecuente en mujeres y en las edades avanzadas de la vida. Hasta en un $80-90 \%$ de los casos se asocia a movimientos periódicos de las piernas. Más del 50\% de los pacientes con este trastorno tienen una historia familiar de síndrome de piernas inquietas; en las formas hereditarias, el inicio de los síntomas es más precoz. Como principales factores etiológicos se han descrito la anemia ferropénica, la insuficiencia renal crónica,

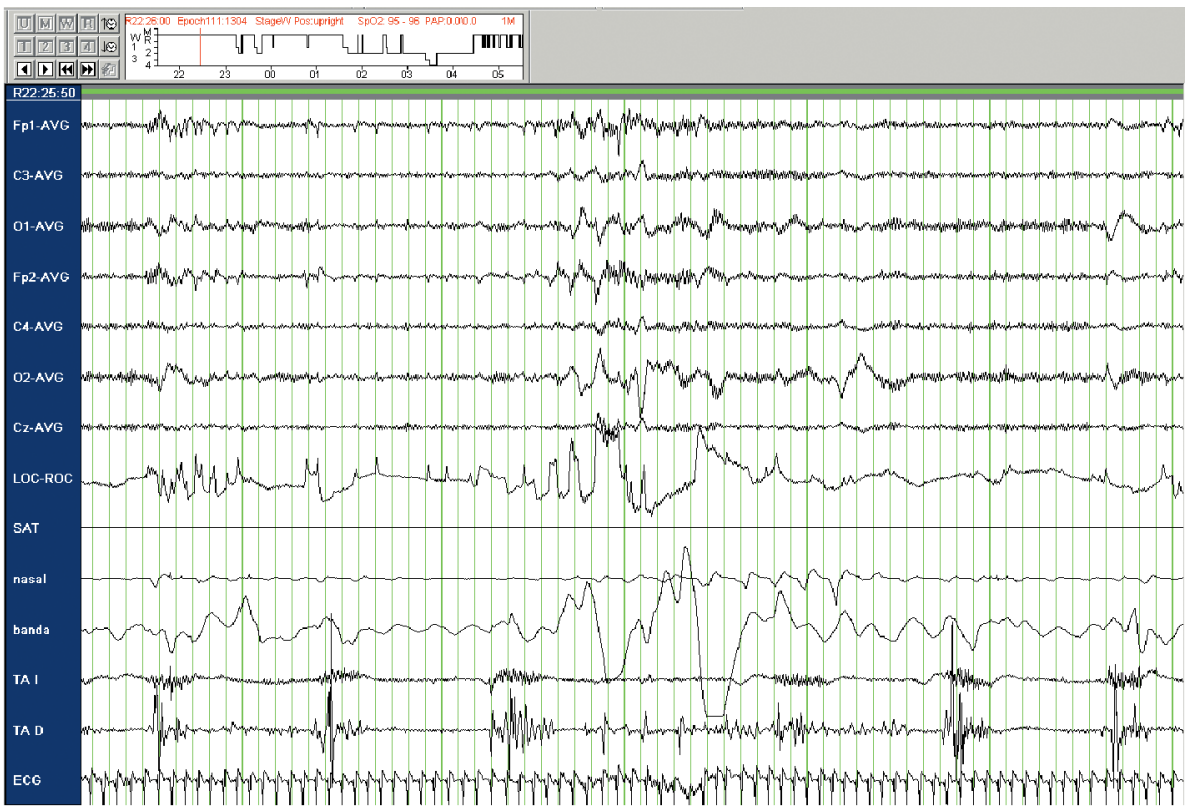

Figura 4. Registro polisomnográfico en piernas inquietas. Está en vigilia (movimientos oculares de vigilia en el electrooculograma LOC-ROC), y se observan movimientos irregulares aunque con cierta periodicidad en ambas piernas (TAD, TAI). La paciente está incorporada (posición upright). 
la diabetes mellitus o la enfermedad de Parkinson; además, algunos fármacos sedantes, antihistamínicos, neurolépticos o antidepresivos pueden agravar los síntomas. El tratamiento debe enfocarse hacia una corrección de la causa (anemia la más frecuente). Cuando ésta no es identificable, puede administrarse tratamiento farmacológico con Clonazepam o agentes dopaminérgicos ${ }^{45,46}$.

Movimientos periódicos de las piernas. Son episodios repetidos de movimientos bruscos, rápidos e involuntarios, de las extremidades ${ }^{47}$. Suelen producirse en secuencias de 4 ó más movimientos, separados entre sí por un intervalo de 5 a 90 segundos (habitualmente 20-40 segundos). Los episodios ocurren sobre todo en las fases 1 y 2 del sueño, pero pueden extenderse a lo largo de toda la noche. Como consecuencia de los mismos, se producen microdespertares repetidos y una interrupción brusca del sueño, que aunque el paciente no sea consciente de ello, desestructuran el patrón normal del sueño. Pueden producirse también, sobre todo cuando los síntomas son más intensos, durante la vigilia, pero no todos los autores están de acuerdo en su relevan$\mathrm{cia}^{30,48,49}$. Habitualmente afectan a las extremidades inferiores, y consisten en una extensión del primer dedo del pie, en combinación con una flexión parcial del tobillo, la rodilla y, en ocasiones, también la cadera. Puede además afectar a los miembros superiores.

Su prevalencia aumenta con la edad, afectando a un $34 \%$ de los sujetos mayores de 60 años. Si bien la mayoría de sujetos que padecen el síndrome de piernas inquietas tienen también movimientos periódicos de las piernas, no suele suceder a la inversa.

Sólo requieren tratamiento los pacientes sintomáticos, pudiendo emplearse benzodiacepinas para reducir los despertares, y agentes dopaminérgicos u opiáceos a dosis bajas ${ }^{50}$.

Calambres nocturnos. Consisten en la contracción involuntaria, súbita, intensa y dolorosa de un músculo o grupos musculares de las extremidades inferiores -habitualmente de la pierna o del pie-, durante el sueño. Pueden precederse en ocasiones de una leve sensación dolorosa. Las contracciones duran desde unos pocos segundos hasta varios minutos, y remiten espontáneamente. No es infrecuente que quede una molestia muscular secuelar tras los mismos. Es un trastorno relativamente frecuente, que afecta a un $15 \%$ de la población. Para aliviar o evitar los síntomas pueden realizarse ejercicios de estiramiento muscular, aplicación de calor local, masoterapia o incluso la movilización de la extremidad. Pueden emplearse también complejos vitamínicos y suplementos electrolíticos.

Bruxismo. Durante el sueño es frecuente que se produzca una contracción de los músculos maseteros, pterigoideos internos y temporales, provocando un cierre enérgico de la mandíbula superior e inferior $^{51,52}$. Estas contracciones pueden ser aisladas y sostenidas, contracción tónica, o pueden cursar como series repetitivas, rítmicas, actividad muscular masticatoria rítmica. Si estas últimas son muy intensas, el golpeteo de las piezas dentales puede producir un ruido o "rechinar", conocido como bruxismo. Ello puede provocar un desgaste del esmalte dental, dolor dental o mandibular, alteraciones de la articulación temporo-mandibular y cefalea. Cuando es intenso puede incluso interrumpir el sueño. Es más frecuente en la infancia, donde su prevalencia es de un 14-17\%, reduciéndose con la edad; en adultos es del 3\%. Para su tratamiento pueden emplearse férulas de protección.

Movimientos rítmicos durante el sueño. Son movimientos rítmicos, estereotipados, como de balanceo de la cabeza o de todo el cuerpo, que a veces se asocian a sonidos guturales, y que suelen emplearse realizarse durante la conciliación del sueño o durante el mismo. Suelen iniciarse a los 9 meses de edad, y que prácticamente desaparecen para los 5 años, por lo que no requieren tratamiento.

\section{SÍNTOMAS AISLADOS}

Se incluyen en este apartado aquellos sujetos que tienen una elevada o baja necesidad de sueño. En el primero de los casos, y desde la infancia, los sujetos necesitan un tiempo total de sueño de 10 ó más horas al 
día, refiriendo somnolencia si no duermen esa cantidad de tiempo. En el segundo de los casos, los sujetos no son capaces de dormir tanto tiempo como la mayoría de las personas, durmiendo habitualmente menos de 5 horas por noche, y a pesar de lo cual no refieren síntomas diurnos.

Ronquido. Es un sonido respiratorio que se genera durante el sueño por el paso del aire en la vía aérea alta, especialmente durante la fase de inspiración aunque también puede ocurrir en la espiración. Puede ser secundario a causas tanto parafisiológicas como patológicas. En este contexto, el ronquido no es sino un síntoma y no causa síntomas de somnolencia diurna ni insomnio.

Somniloquios. Consisten en hablar, desde palabras aisladas hasta discursos completos, y con grados variables de comprensión, durante el sueño. Se trata de un fenómeno inocuo, del cual el sujeto no recuerda nada al día siguiente.

Mioclonías del sueño. Son contracciones simultáneas, cortas y súbitas, del cuerpo o de una o más partes del cuerpo que ocurren al inicio del sueño. Habitualmente son una contracción única y asimétrica, y con frecuencia cursan con la impresión subjetiva de caída.

Mioclonías benignas de la infancia. Son movimientos mioclónicos repetidos que ocurren durante el sueño en la infancia. Con frecuencia son bilaterales y masivos, incluyendo grandes grupos musculares ${ }^{53}$.

Otros trastornos. También es posible encontrar de forma aislada son el temblor hipnagógico de las piernas, en los que el sujeto presenta durante la transición de la fase de vigilia a la de sueño, o en las fases 1 y 2 , un movimiento tremórico rítmico de los pies o tobillos; y las mioclonías propioespinales, que consisten en sacudidas mioclónicas de la musculatura axial, y que también ocurren con mayor frecuencia durante la transición vigilia-sueño.

\section{OTROS TRASTORNOS DEL SUEÑO}

Incluye todos aquellos trastornos que no pueden ser clasificados en ninguna de las categorías anteriores de la segunda Clasificación Internacional de los Trastornos del Sueño. Son varias las razones por las que un trastorno del sueño puede clasificarse en este grupo: 1. el trastorno del sueño cumple características de más de una de las anteriores categorías; 2 . no hay datos suficientes para establecer un diagnóstico dentro de las anteriores categorías. Probablemente, mientras siga en curso la segunda Clasificación Internacional de los Trastornos del Sueño, se descubrirán y describirán nuevos trastornos del sueño, que se englobarán en esta categoría hasta que se establezca un diagnóstico definitivo en siguientes ediciones.

\section{APÉNDICE}

Apéndice A. Este apéndice contiene un pequeño listado de enfermedades médicas que afectan al sueño, o que se ven afectadas por el sueño, y que son de particular importancia en las Unidades de Sueño.

Insomnio Familiar Fatal. Es una enfermedad priónica que se caracteriza por el desarrollo progresivo de trastorno del sueño (insomnio, somnolencia diurna y estupor onírico), hiperactividad autonómica (pirexia, sialorrea, hiperhidrosis, taquicardia, taquipnea,...), disfagia, disartria, temblor, mioclonías espontáneas y reflejas, posturas distónicas, trastorno de la marcha y signo de Babinski. La enfermedad evoluciona hacia el coma y la muerte en unos pocos meses ${ }^{54,55}$.

Otras entidades incluidas en este apéndice son la fibromialgia, algunos síndromes epilépticos que se manifiestan sólo o predominantemente durante el sueño (epilepsia nocturna del lóbulo frontal, epilepsia benigna de la infancia con puntas centrotemporales, epilepsia occipital de la infancia de inicio temprano o tardío, epilepsia juvenil mioclónica, crisis tónico-clónicas generalizadas del despertar, un subtipo de epilepsia del lóbulo temporal, el síndrome de Landau-Kleffner y el síndrome de puntas-ondas continuas durante el sueño no REM), diferentes tipos de cefaleas que se desarrollan durante el sueño o al despertarse (migraña, cefalea en acúmulos, hemicránea paroxística, cefalea hípnica, y cefaleas secundarias a hipertensión intracraneal, hipertensión arterial, etc.), el reflujo gas- 
troesofágico, la angina de Prinzmetal y el laringoespasmo nocturno ${ }^{25,56,57}$.

Apéndice B. Incluye aquellos trastornos psiquiátricos y del comportamiento que plantean con frecuencia el diagnóstico diferencial con los trastornos del sueño, y que se resumen en la DSM-IV-TR.

\section{CONSIDERACIONES FINALES}

En este trabajo hemos revisado la clasificación más reciente de los trastornos del sueño. Aunque los grupos parecen bien diferenciados, es frecuente la asociación de trastornos y es posible la clasificación de algunos de ellos en varios grupos. Esta clasificación no es por tanto definitiva, pero sí facilita el estudio y comprensión de estos trastornos, así como la comunicación científica. Tomándola como una ayuda y no como algo definitivo será más útil para los clínicos.

\section{BIBLIOGRAFÍA}

1. The International Classification of Sleep Disorders. Revised. Diagnostic and coding manual. 2nd. Rochester, Minnesota: American Sleep Disorders Association; 1997.

2. AASM. The International Classification of Sleep Disorders. Diagnositc and coding manual. 2. Westchester, IL: AASM; 2005.

3. International Classification of Diseases, Ninth Revision (ICD-9). Ginebra: OMS; 1977.

4. International Statistical Classification of Diseases and Related Health Problems, 10th Revision. Ginebra: OMS; 1992.

5. ThORPY MJ. Classification of sleep disorders. J Clin Neurophysiol 1990; 7: 67-81.

6. National Institutes of Health State of the Science Conference statement on Manifestations and Management of Chronic Insomnia in Adults. Sleep 2005; 28: 10491057.

7. Summers MO, CRisostomo MI, Stepanski EJ. Recent developments in the classification, evaluation, and treatment of insomnia. Chest 2006; 130: 276-286.

8. Sateia MJ, Doghramui K, Hauri PJ, Morin CM. Evaluation of chronic insomnia. An American Academy of Sleep Medicine review. Sleep 2000; 23: 243-308.

9. KRYSTAL AD. The changing perspective on chronic insomnia management. J Clin Psychiatry 2004; 65 Suppl 8: 20-25.
10. CoHen AS, KAUBE H. Rare nocturnal headaches. Curr Opin Neurol 2004; 17: 295299.

11. Ware JC, Morewitz J. Diagnosis and treatment of insomnia and depression. J Clin Psychiatry 1991; 52 Suppl: 55-61.

12. EDINGER JD, KRYSTAL AD. Subtyping primary insomnia: is sleep state misperception a distinct clinical entity? Sleep Med Rev 2003; 7: 203-214.

13.Espie CA, BRoomfield NM, MacMahon KM, MACPHEE LM, TAYLOR LM. The attentionintention-effort pathway in the development of psychophysiologic insomnia: a theoretical review. Sleep Med Rev 2006; 10: 215-245.

14. Perlis ML, Giles DE, Mendelson WB, Bootzin RR, WYATT JK. Psychophysiological insomnia: the behavioural model and a neurocognitive perspective. J Sleep Res 1997; 6: 179-188.

15. AtTARIAN HP, Duntley S, Brown KM. Reverse sleep state misperception. Sleep Med 2004; 5: 269-272.

16. Abad VC, Guilleminault C. Neurological perspective on obstructive and nonobstructive sleep apnea. Semin Neurol 2004; 24: 261-269.

17. Costa Orvay JA, Pons OM. Ondine syndrome: diagnosis and management. An Pediatr (Barc ) 2005; 63: 426-432.

18. GiBSON GJ. Obstructive sleep apnoea syndrome: underestimated and undertreated. Br Med Bull 2005; 72:49-65. Print; 2004. 49-65.

19. PhilliPSon EA. Sleep apnea. A major public health problem. N Engl J Med 1993; 328: 1271-1273.

20. YAgGi HK, Concato J, KeRnan WN, Lichtman JH, Brass LM, MoHSEnIN V. Obstructive sleep apnea as a risk factor for stroke and death. N Engl J Med 2005; 353: 2034-2041.

21. Morgenthaler TI, Kagramanov V, Hanak V, DECKER PA. Complex sleep apnea syndrome: is it a unique clinical syndrome? Sleep 2006; 29: 1203-1209.

22. Flemons WW. Clinical practice. Obstructive sleep apnea. N Engl J Med 2002; 347: 498-504.

23. Young TJ, Silber MH. Hypersomnias of central origin. Chest 2006; 130: 913-920.

24. BAumANn CR, BASSETti CL. Hypocretins (orexins) and sleep-wake disorders. Lancet Neurol 2005; 4: 673-682. 
25. Evers S, GoAdsby PJ. Hypnic headache: clinical features, pathophysiology, and treatment. Neurology 2003; 60: 905-909.

26. Arnulf I, Zeitzer JM, File J, Farber N, Mignot E. Kleine-Levin syndrome: a systematic review of 186 cases in the literature. Brain 2005; 128: 2763-2776.

27. Mahowald MW, Bornemann MC, Schenck CH. Parasomnias. Semin Neurol 2004; 24: 283292.

28. Hublin C, KAPRIO J. Genetic aspects and genetic epidemiology of parasomnias. Sleep Med Rev 2003; 7: 413-421.

29. Mahowald MW, SchENCK CH. Non-rapid eye movement sleep parasomnias. Neurol Clin 2005; 23: 1077-1106.

30. LeE MK, Guilleminault C. Rapid Eye Movement Sleep-related Parasomnias. Curr Treat Options Neurol 2002; 4: 113-120.

31. Schenck CH, Mahowald MW. Rapid eye movement sleep parasomnias. Neurol Clin 2005; 23: 1107-1126.

32. Gagnon JF, Postuma RB, Mazza S, Doyon J, MonTPLAISIR J. Rapid-eye-movement sleep behaviour disorder and neurodegenerative diseases. Lancet Neurol 2006; 5: 424-432.

33. Fantini ML, Ferini-Strambi L, Montplaisir J. Idiopathic REM sleep behavior disorder: toward a better nosologic definition. Neurology 2005; 64: 780-786.

34. SCHENCK CH, MAHOWALD MW. REM sleep behavior disorder: clinical, developmental, and neuroscience perspectives 16 years after its formal identification in sleep. Sleep 2002; 25: 120-138.

35. Boeve BF, Silber MH, Ferman TJ, Lucas JA, PARISI JE. Association of REM sleep behavior disorder and neurodegenerative disease may reflect an underlying synucleinopathy. Mov Disord 2001; 16: 622-630.

36. Iranzo A, SANTAMaria J, Rye DB, VAlLdeoriola F, MARTI MJ, MunOz E et al . Characteristics of idiopathic REM sleep behavior disorder and that associated with MSA and PD. Neurology 2005; 65: 247-252.

37. Iriarte J, Alegre M, Urrestarazu E, Viteri C, ARcocha J, ARTIEDA J. Continuous positive airway pressure as treatment for catathrenia (nocturnal groaning). Neurology 2006; 66: 609-610.

38. Lugaresi E, Provini F. Sleep and movement disorders. Neurol Sci 2005; 26 Suppl 3: 173175.
39. Phillips B. Movement disorders: a sleep specialist's perspective. Neurology 2004; 62 : 9-16.

40. JANKOVIC J. International Classification of Diseases, tenth revision: neurological adaptation (ICD-10 NA): extrapyramidal and movement disorders. Mov Disord 1995; 10: 533-540.

41. Brotini S, GigLi GL. Epidemiology and clinical features of sleep disorders in extrapyramidal disease. Sleep Med 2004; 5: 169-179.

42. WALTERS AS. Toward a better definition of the restless legs syndrome. The International Restless Legs Syndrome Study Group. Mov Disord 1995; 10: 634-642.

43. AlLEN RP, EARLEy CJ. Restless legs syndrome: a review of clinical and pathophysiologic features. J Clin Neurophysiol 2001; 18: 128147.

44. Allen RP, Picchietti D, Hening WA, Trenkwalder C, WAlters AS, Montplaisi J. Restless legs syndrome: diagnostic criteria, special considerations, and epidemiology. A report from the restless legs syndrome diagnosis and epidemiology workshop at the National Institutes of Health. Sleep Med 2003; 4: 101-119.

45. Clavadetscher SC, Gugger M, Bassetti CL. Restless legs syndrome: clinical experience with long-term treatment. Sleep Med 2004; 5 : 495-500.

46. García-Borreguero D, Odin P, Schwarz C. Restless legs syndrome: an overview of the current understanding and management. Acta Neurol Scand 2004; 109: 303-317.

47. Chabli A, Michaud M, Montplaisir J. Periodic arm movements in patients with the restless legs syndrome. Eur Neurol 2000; 44: 133-138.

48. Haba-Rubio J, Staner L, Krieger J, Macher JP. What is the clinical significance of periodic limb movements during sleep? Neurophysiol Clin 2004; 34: 293-300.

49. Karadeniz D, Ondze B, Besset A, Billiard M. Are periodic leg movements during sleep (PLMS) responsible for sleep disruption in insomnia patients? Eur J Neurol 2000; 7: 331336.

50. SCHENCK CH. Restless legs syndrome and periodic limb movements of sleep: global therapeutic considerations. Sleep Med Rev 2002; 6: 247-251.

51. BADER G, LAVIGNE G. Sleep bruxism; an overview of an oromandibular sleep movement disorder. Review article. Sleep Med Rev 2000; 4: 27-43. 
52. De LaAt A, Macaluso GM. Sleep bruxism as a motor disorder. Mov Disord 2002; 17 Suppl 2: S67-S69.

53. Pachatz C, Fusco L, Vigevano F. Benign myoclonus of early infancy. Epileptic Disord 1999; 1: 57-61.

54. Lugaresi E, Tobler I, Gambetti P, Montagna P. The pathophysiology of fatal familial insomnia. Brain Pathol 1998; 8: 521-526.
55. Montagna P, Gambetti P, Cortelli P y Lugaresi E. Familial and sporadic fatal insomnia. Lancet Neurol 2003; 2: 167-176.

56. Derry CP, Duncan JS, Berkovic SF. Paroxysmal motor disorders of sleep: the clinical spectrum and differentiation from epilepsy. Epilepsia 2006; 47: 1775-1791.

57. FoldVARY-SchaEFER N, GRIGG-DAMBERGER M. Sleep and epilepsy: what we know, don't know, and need to know. J Clin Neurophysiol 2006; 23: 4-20. 